\title{
Hedgehog-mediated regulation of PPAR $\gamma$ controls metabolic patterns in neural precursors and shh-driven medulloblastoma
}

\author{
Bobby Bhatia $\cdot$ Chad R. Potts $\cdot$ Cemile Guldal $\cdot$ \\ SunPhil Choi $\cdot$ Andrey Korshunov $\cdot$ Stefan Pfister · \\ Anna M. Kenney • Zaher A. Nahlé
}

Received: 10 October 2011/Revised: 27 February 2012/Accepted: 29 February 2012/Published online: 11 March 2012

(C) The Author(s) 2012. This article is published with open access at Springerlink.com

\begin{abstract}
Sonic hedgehog (Shh) signaling is critical during development and its aberration is common across the spectrum of human malignancies. In the cerebellum, excessive activity of the Shh signaling pathway is associated with the devastating pediatric brain tumor medulloblastoma. We previously demonstrated that exaggerated de novo lipid synthesis is a hallmark of Shh-driven medulloblastoma and that hedgehog signaling inactivates the Rb/E2F tumor suppressor complex to promote lipogenesis. Indeed, such Shh-mediated metabolic reprogramming fuels tumor
\end{abstract}

Electronic supplementary material The online version of this article (doi:10.1007/s00401-012-0968-6) contains supplementary material, which is available to authorized users.

\section{B. Bhatia $\cdot$ C. Guldal}

Department of Cancer Biology and Genetics,

Memorial Sloan-Kettering Cancer Center,

New York, NY, USA

C. R. Potts · S. Choi - A. M. Kenney - Z. A. Nahlé

Department of Neurological Surgery, Vanderbilt University

Medical Center, Nashville, TN, USA

A. M. Kenney · Z. A. Nahlé $(\bowtie)$

Department of Cancer Biology, Vanderbilt University Medical

Center, Nashville, TN, USA

e-mail: zaher.nahle@vanderbilt.edu

A. Korshunov

Clinical Cooperation Unit Neuropathology, German Cancer

Research Center (DKFZ), Heidelberg, Germany

\section{S. Pfister}

Division of Molecular Genetics, German Cancer Research

Center (DKFZ), Heidelberg, Germany

Present Address:

B. Bhatia

Millipore Corporation, Billerica, MA, USA progression, in an E2F1- and FASN-dependent manner. Here, we show that the nutrient sensor PPAR $\gamma$ is a key component of the Shh metabolic network, particularly its regulation of glycolysis. Our data show that in primary cerebellar granule neural precursors (CGNPs), proposed medulloblastoma cellsof-origin, Shh stimulation elicits a marked induction of PPAR $\gamma$ alongside major glycolytic markers. This is also documented in the actively proliferating Shh-responsive CGNPs in the developing cerebellum, and PPAR $\gamma$ expression is strikingly elevated in Shh-driven medulloblastoma in vivo. Importantly, pharmacological blockade of PPAR $\gamma$ and/or $\mathrm{Rb}$ inactivation inhibits CGNP proliferation, drives medulloblastoma cell death and extends survival of medulloblastomabearing animals in vivo. This coupling of mitogenic Shh signaling to a major nutrient sensor and metabolic transcriptional regulator define a novel mechanism through which Shh signaling engages the nutrient sensing machinery in brain cancer, controls the cell cycle, and regulates the glycolytic index. This also reveals a dominant role of Shh in the etiology of glucose metabolism in medulloblastoma and underscores the function of the Shh $\rightarrow$ E2F1 $\rightarrow$ PPAR $\gamma$ axis in altering substrate utilization patterns in brain cancers in favor of tumor growth. These findings emphasize the value of PPAR $\gamma$ downstream of Shh as a global therapeutic target in hedgehogdependent and/or $\mathrm{Rb}$-inactivated tumors.

Keywords Medulloblastoma $\cdot$ Cerebellum $\cdot$ Metabolism . $\operatorname{PPAR} \gamma \cdot$ Glycolysis $\cdot$ Sonic hedgehog · Cancer .

Tumor metabolism

\section{Introduction}

Medulloblastomas (MBs) remain the leading cause of death from solid cancers in children [30]. These predominantly 
pediatric tumors of cerebellar origin present in infants, children, and to a lesser extent in the adult population. Concordantly, MBs are associated with aberrations in signaling cascades critical for brain development, organ patterning, and cell-fate determination like those controlled by the Wnt (WNT) or the hedgehog (HH) pathway. As high as $30 \%$ of total human MBs bear evidence of aberrant hedgehog pathway activation. In this context, loss of function mutations in Patched (Ptch) or activating mutations in Smoothened (Smo), both trans-membrane proteins and essential components of Shh signaling, have been identified in human medulloblastomas [32, 34, 37, 48, 49]. Mutations in cytoplasmic components of the hedgehog pathway such as Suppressor of Fused ( $\mathrm{SuFu}$ ) have also been described [42]. Importantly, ectopic activation of Shh signaling [35, 36], constitutive activation of Smo itself $[14,15]$, or Ptch loss-of-heterozygosity [10] are all sufficient, independently, to drive medulloblastoma formation in vivo. Consistently, global inhibition of the Shh pathway at the level of its receptors, while transiently beneficial in $\mathrm{MB}$ patients, remains confounded by a rapid development of drug resistance, a trait also conserved in hedgehog-associated tumorbearing mice [50]. Understanding the complexity of Shh signaling and the corresponding oncogenic network downstream of hedgehog is as such critical for developing effective and specific nodes for therapeutic manipulation.

In a recent study, we have demonstrated that hedgehogdriven MBs are distinguished by a dramatic accumulation of neutral lipids, underscoring these tumors' metabolic, markedly lipogenic nature [2]. Such lipogenic features are similar to those described in gliomas with EGF receptor mutations [11] or a number of other human malignancies including cancer of the breast, lung, and prostate [25]. Importantly, we demonstrated mechanistically that the effect of Shh on lipogenic metabolism proceeds through a multi-step program involving the inactivation of the tumor suppressor $\mathrm{Rb}$ and the induction of its negative target E2F1, a key transcriptional regulator of proliferation networks [16, 27, 47]. Indeed, Shhdependent induction of E2F1 promotes de novo lipid synthesis, induces the key enzyme Fatty Acid synthase (FASN), and primes the lipogenic machinery for a bioenergetics environment conducive to rapid proliferation. Impairing E2F activity and/or targeting de novo lipid synthesis with pharmacological means produced effective, reproducible therapeutic outcomes in MBs-bearing animals in vivo. These non-invasive approaches are especially promising for current $\mathrm{MB}$ treatments that damage the still-developing brain and result in catastrophic life-long side effects [31]. It will be insightful to decipher the key components regulating the metabolic machinery, in particular, those promoting the acquisition of permissive substrate utilization patterns that fuel disease progression and enable malignant transformation.
It is widely established that adaptations to perceived stress conditions are hallmarks of proper functioning in systemic physiology. In fact, reciprocity in coordinated pathways of glucose and lipid metabolism and flexible metabolic shifts are common with dietary flux conditions including starvation, fasting or postprandial states [39-41]. Such exquisite capacity for nutrient sensing is attributed, broadly speaking, to a complex web of signaling events. This includes (i) intracellular messengers like cAMP and associated kinases, (ii) mitochondrial enzymes responsive to acute changes in biochemical ratios such acetyl-CoA/ $\mathrm{CoA}$ and NADH/NAD+ ratios, (iii) hormones that recruit specific substrate receptors like Glut4, and/or (iv) transcription factors that could alters metabolic patterns and substrate utilization at multiple, programmatic levels. A particular class of transcription factors with such capacity for reprogramming cellular responses and dictating nutrient sensing and signaling events are the fatty-acid activated peroxisome proliferator-activated receptors (PPARs). PPARs can elicit chronic, sustainable metabolic shifts through their transcriptional effects on metabolic enzyme gene expression. It is established that PPAR $\gamma$ activation confers significant benefit and ameliorate insulin sensitivity in vivo and ex vivo.

Here, we demonstrate the existence of a novel mechanism connecting Shh signaling to the PPAR $\gamma$ transcriptional machinery in neural precursors and in Shhdriven medulloblastoma. We show that Shh recruits the steroid nuclear receptor $\operatorname{PPAR} \gamma$, a key element in the nutrient sensing network, to reprogram cellular metabolism including the regulation of glycolysis proper. Indeed, Shhdependent control of PPAR $\gamma$ dramatically activates the glycolytic modulators Glut4, hexokinase II (HKII) and pyruvate kinase M2 (PKM2), and promote glucose uptake in tumors in vivo. Importantly, the mechanism linking Shh to PPAR $\gamma$ and its aforementioned targets requires E2F1 in vivo and ex vivo. In addition, this process is fully reversible with antagonizing $\operatorname{PPAR} \gamma$, an event that remarkably blunts tumor proliferation and extends survival of animals with medulloblastoma in vivo. In short, findings of this study provide critical insights into how vital and interlocked metabolic networks are altered or co-opted in Shhdriven MBs. The data also underscore the significant role of PPAR $\gamma$ downstream of Shh in dictating modes of substrate utilization and defining metabolic patterns in these tumors, and possibly other hedgehog-associated cancers. Given the fundamental role for PPAR $\gamma$ in nutrient sensing and the integration of dietary signals on the one hand and Shh functions in a myriad of biological activities like proliferation and self-renewal on the other hand, such link highlights the interplay between cell cycle regulators and the fuel processing machinery. Furthermore, these results are equally relevant to the pathophysiology of chronic 
diseases where glucose metabolism is dysregulated, particularly where sonic hedgehog activities are also implicated [21,22]. Finally, considering the central role of the $\mathrm{Rb}-\mathrm{E} 2 \mathrm{~F}$ complex in survival and proliferation and the widespread $\mathrm{Rb}$ pathway mutations found in human cancers, our findings could assist in better defining the etiology of tumor metabolism, in particular the perplexing phenotype of aerobic glycolysis and the lack of efficient mitochondrial oxidation of glucose despite the ostensible need for ATP in hyperproliferative cells. The requirement for E2F1 in Shhregulated PPAR $\gamma$ dependent-glycolysis, as demonstrated, highlights its engagement in metabolic core fluxes and underscores the critical role for the $\mathrm{Rb} / \mathrm{E} 2 \mathrm{~F}$ complex in metabolic reprogramming of medulloblastomas and their cells-of-origin.

\section{Materials and methods}

Animal studies

Wild type C57-BL6 mice and NeuroD2-SmoA1 mice bearing tumors were administered $10 \%$ dimethyl sulfoxide (DMSO, control), olomoucine (Calbiochem) at $6 \mathrm{mg} / \mathrm{kg}$ daily or GW9662 (Cayman Chemicals) at $10 \mathrm{mg} / \mathrm{kg}$ daily by intraperitoneal (i.p.) injection. Wild-type C57/BL6 mice, NeuroD2-SmoA1 mice, and E2F1-null mice were obtained from Jackson Laboratory (Bar Harbor, ME, USA) and typically were 4-5 months of age when demonstrating the signs of a tumor (ataxia, etc.).

\section{Cell culture}

Cerebellar granule neural precursor cultures were generated as previously described in detail [19]. The cell culture media consisted of DMEM-F12 (Gibco, Grand Island, NY) and $1 \times$ dilutions of $\mathrm{N} 2$ supplement (as $100 \times$ from Gibco), Penicillin Streptomycin $(100 \times$ from Gibco) and potassium chloride (2.5 M is a $100 \times$ stock). P4 and P5 wild type C57/ BL6 pups were killed and the cerebellum extracted and placed into $1 \times$ Hanks buffered saline solution (HBSS) (Gibco) with $6 \mathrm{gm} / \mathrm{L}$ of glucose at a $\mathrm{pH}$ of 7.4. The HBSS was aspirated under a tissue hood and trypsin (Gibco) was added with $1 \mathrm{mg} / \mathrm{ml}$ of DNAse. The trypsin was then inactivated with the media containing $10 \% \mathrm{FCS}$ and the cerebella titurated twice. Following a $5 \mathrm{~min}$ spin at $1,500 \mathrm{rpm}$, the pellet was resuspended in $10 \%$ FCS media. Cells were then plated on polyornithine-coated six well plates at a concentration of 3 million cells $/ \mathrm{mL}$ in $1 \mathrm{~mL}$ of the $10 \%$ FCS media for $6-12 \mathrm{~h}$. The media were then replaced with FCS free media and given their treatments and incubated for $24 \mathrm{~h}$ before protein lysis. Where indicated Shh (R\&D Systems, Minneapolis, MN, USA) was used at a concentration of $3 \mu \mathrm{g} / \mathrm{ml}$, cyclopamine (R\&D Systems) was used at $1 \mu \mathrm{g} / \mathrm{ml}$ and GW9662 (Cayman Chemical, Ann Arbor, MI, USA) was used at 50 or $100 \mathrm{nM}$.

\section{Western blotting}

Protein extracts were prepared as previously described [20]. A total of $40 \mu \mathrm{g}$ of murine cerebellum, medulloblastoma, or CGNP protein extract were run on $8-12 \%$ SDS-polyacrylamide gels and transferred to a PVDF membrane (Millipore, Billerica, MA, USA). The blots were blocked and incubated with primary antibodies in $5 \%$ milk in Tris-buffered saline-Tween (TBS-T) overnight in $4{ }^{\circ} \mathrm{C}$. Blots were washed three times and incubated with secondary antibodies in $5 \%$ milk in TBS-T for $2 \mathrm{~h}$ in room temperature. After washing, the signals were developed using the enhanced chemiluminescence method (GE Healthcare, Piscataway, NJ, USA) and the membranes were exposed to Kodak Biomax film. Primary antibodies were: E2F1 (Cell Signaling, Danvers, MA, USA), PPAR $\gamma$ (E-8; Santa Cruz Biotechnology, Santa Cruz, CA, USA), FASN (Cell Signaling), Hexokinase I (Cell Signaling), Hexokinase II (Cell Signaling), Glut4 (Cell Signaling), PKM2 (Cell Signaling), Cyclin D2 (M-20; Santa Cruz) and $\beta$-tubulin (Sigma). Horseradish peroxidase-conjugated secondary antibodies were: goat anti-rabbit $\operatorname{IgG}(\mathrm{H}-\mathrm{L}$; Thermo Scientific, Rockford, IL, USA) and donkey antimouse IgG (H-L; Jackson Immuno-Research, West Grove, PA, USA). Primary antibodies were diluted to 1:1000 for incubation in $5 \%$ milk in TBS-T with the exception of $\beta$-tubulin, which was 1:5,000 while the secondary antibodies were at $1: 10,000$ as recommended by their manufacturers.

Lentivirus production and infection

E2F1 shRNA and scrambled control lentivirus constructs were obtained from The RNAi Consortium (Sigma, St Louis MO). Each construct was transfected into the Pzp53med cell line, which was derived from a medulloblastoma arising in a $\mathrm{Ptc}^{+-} / \mathrm{p} 53^{-/-}$mouse [1], and western blotting was used to determine which shRNAs effectively and specifically targeted E2F1. These constructs were used to prepare lentiviruses. 293T cells grown in 10 \% FBS Dulbecco's modified Eagle's medium (Gibco) were transfected with E2F1 or scrambled control shRNA lentiviral vectors and MISSION lentiviral packaging mix (Sigma) using Fugene 6 in serum-free OPTI-MEM medium (Invitrogen, Carlsbad, CA, USA). Lentiviral supernatants were collected 48 -h post-transfection and filtered through a $0.45 \mu$ filter, then pooled. For infection, Shh-treated CGNPs were exposed to the lentiviral supernatant for $4 \mathrm{~h}$. Control scrambled shRNA lentivirus constructs were used 
to determine specificity for E2F1 and to rule out off-target effects or non-specific results because of the process of virus infection. The lentiviruses were aspirated and replaced with serum-free CGNP medium (above) containing Shh. The cells were lysed 48 -h post-infection.

\section{Immunostaining}

Tissues were fixed in $4 \%$ para-formaldehyde and paraffinembedded as previously described [3]. After dehydration steps and sectioning, tissue slides were washed with $1 \times$ phosphate buffered saline (PBS) and permeabilized in $1 \%$ TritonX-100 for 5 min. Slides were then blocked in $5 \%$ goat serum in PBS-Tween $(1 \times$ PBS and $0.1 \%$ TritonX-100) for $1 \mathrm{~h}$ in room temperature, washed once with $1 \times \mathrm{PBS}$, and incubated with primary antibody in $2.5 \%$ goat serum (in PBSTween) overnight at $4{ }^{\circ} \mathrm{C}$. They were washed three times with $1 \times$ PBS and incubated with secondary antibody for $2 \mathrm{~h}$ at room temperature, then washed and mounted in 40-6-diamidino-2-phenylindole-containing mounting medium (Vector Labs, Burlingame, CA, USA). Primary antibodies used were: E2F1 (H-137; Santa Cruz), PPAR $\gamma$ (E-8; Santa Cruz), FASN (Cell Signaling, Danvers MA), Hexokinase I (Cell Signaling), Hexokinase II (Cell Signaling), Glut4 (Cell Signaling), PKM2 (Cell Signaling), P-Histone H3 (Cell Signaling), and Ki67 (Vector Labs, Burlingame CA). Secondary fluorescent-tagged antibodies were: Alexa Fluor goat anti-rabbit 488/594 (Invitrogen, Carlsbad, CA) and Alexa Fluor goat anti-mouse 488/594 (Invitrogen).

\section{Image capturing}

Immunostaining performed on tissue sections was visualized using a Leica DM5000B microscope and images were captured with Leica FW400 software (Leica Microsystems, Bannockburn, IL, USA).

\section{Oil Red O staining}

Oil Red O staining of neutral lipids in NeuroD2-SmoA1 medulloblastoma frozen sections was performed by the histology core in the Department of Pathology and Laboratory Medicine in the Hospital for Special Surgery and by the microcytometry core facility at Memorial Sloan-Kettering Cancer Center according to established protocols.

\section{PET scanning}

For fluorine-18 fluoro-deoxyglucose (FDG) studies using the Focus 120 microPET small-animal PET scanner (Concorde Microsystems, Knoxville, TN), animals were fasted for $6 \mathrm{~h}$ prior to injection of the radiotracer; access to water was permitted. Mice were bolus injected via lateral tail vein with $300 \mathrm{mCi}$ of the PET radiotracer FDG in $\sim 200 \mathrm{ml}$ of normal saline. At $\sim 45$-min post-injection, the mouse was first anesthetized by inhalation of a $2 \%$ isofluorane/oxygen gas mixture (flow rate of $2 \mathrm{~L} / \mathrm{min}$.) and placed on the scanner bed in the prone position. The acquisition is set up for $\sim 5$ min using a photopeak energy window of $350-750 \mathrm{keV}$, and a coincidence timing window of $6 \mathrm{nsec}$-and launched using the microPET's microPET Manager ${ }^{\mathrm{TM}}$ computer software. Data are acquired in list mode. The resulting data are sorted into 2D histograms by Fourier re-binning and transverse images are reconstructed by filtered back-projection (FBP) into a 3D imaging matrix using the system Nyquist frequency as the cut-off. The image data are normalized to correct for nonuniformity of response of the microPET, dead-time count losses, positron branching ratio, and physical decay to the time of injection, attenuation, scatter, or partial-volume averaging corrections are generally not applied. An empirically determined system calibration factor (in units of $\mathrm{mCi} / \mathrm{mL} / \mathrm{cps} /$ voxel) for mice is used to convert voxel count rates to activity concentrations, and the resulting image data are then normalized to the administered activity to parameterize images in terms of percent of the injected doses per gram (\% ID/gm). Manually drawn 2D regions-ofinterest (ROIs) or 3D volumes-of-interest (VOIs) are used to determined the mean and maximum \%ID/g (decay corrected to the time of injection) in tumors and various organs using ASIPro VM ${ }^{\mathrm{TM}}$ computer software (Concorde Microsystems).

\section{Cell viability}

Cell viability was determined with the cell Titer Glo assay (Promega, Madison, WI) which utilizes luminescent reagents reacting with ATP yielding a quantitative expression of living cells in the sample expressed as a relative luminescence between samples. This luminescence was measured with the Glo Max Multi + (Promega) plate reader. Statistical analysis was accomplished with SAS software package (SAS Inst. Inc., N.C., USA). Significant differences between mean were determined by ANOVA procedure test and a $P$ value of $<0.05$ was considered to be statistically significant. The results are expressed as the mean $\pm \mathrm{SD}$ from quadruplicate test data.

\section{Results}

Marked deregulation of PPAR $\gamma$ in Shh-driven mouse medulloblastomas

We have previously reported that the subclass of medulloblastomas resulting from aberrant activation of Shh 
signaling $[14,15]$ is marked by strikingly high levels of de novo fatty acid synthesis, as determined by staining for neutral lipids (Fig. 1a, top left, Oil Red O staining), compared to the adjacent non-tumorigenic cerebellar tissue (bottom left). This exaggerated lipogenesis is associated with high levels of proliferation as indicated by Ki67 immunofluorescence, and increased cdk activity, leading to $\mathrm{Rb}$ inactivation and induction of E2F1 (Fig. 1a, b). Indeed,
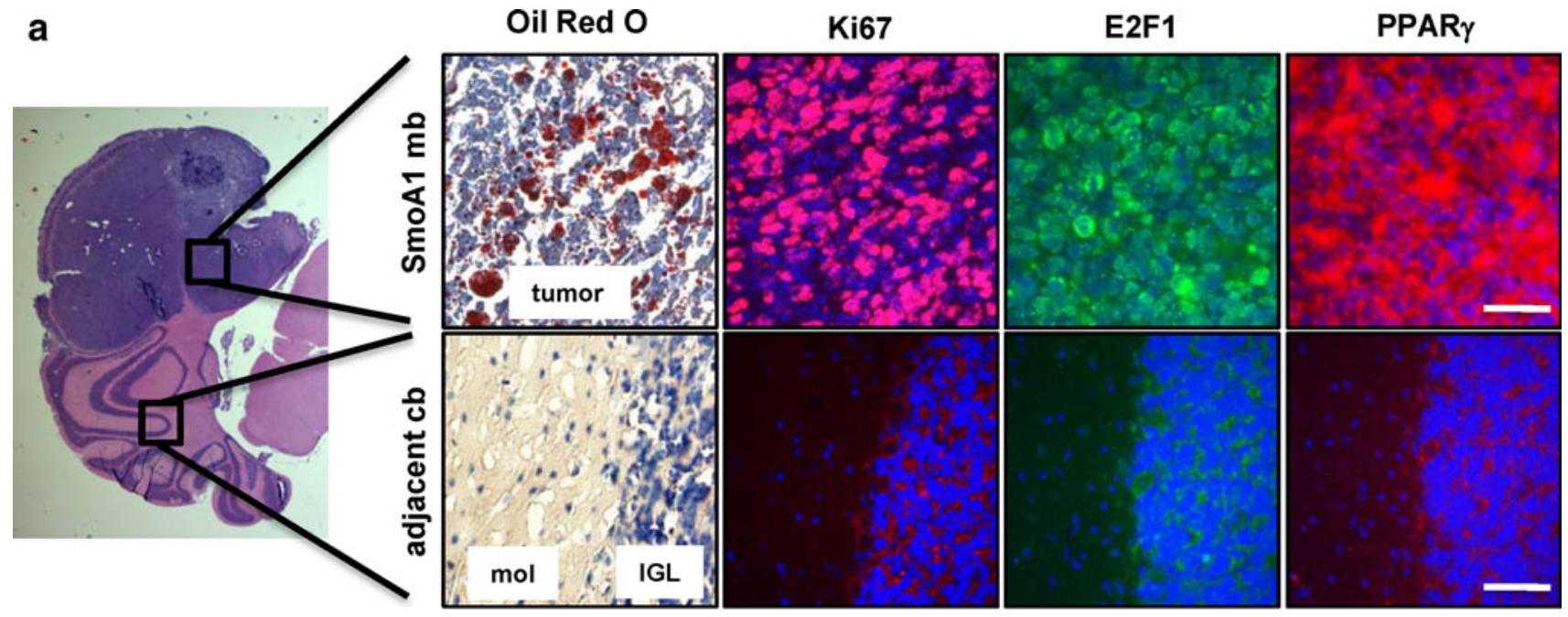

b

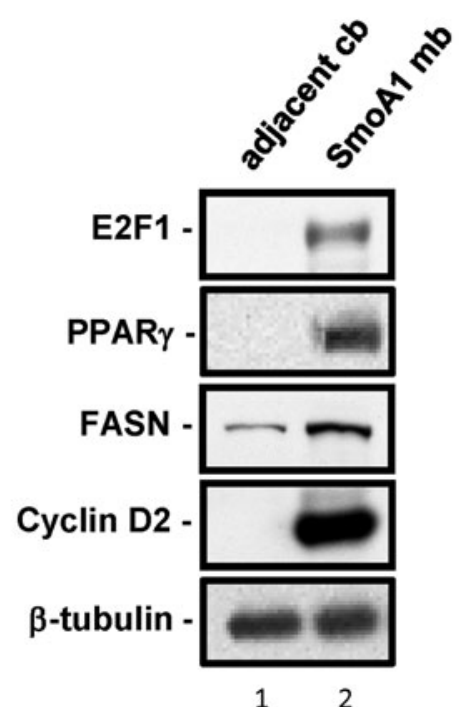

Fig. 1 Shh-induced mouse medulloblastomas have robust lipogenesis, increased levels of E2F1 and PPAR $\gamma$ and cell proliferation. a Triglyceride accumulation was analyzed in a NeuroD2-SmoA1 medulloblastoma using Oil Red O staining; neutral lipids appear as red droplets. NeuroD2-SmoA1 medulloblastoma (top row) and adjacent non-tumorous cerebellum (bottom row) were subjected to immunofluorescence analyses for $\mathrm{E} 2 \mathrm{~F} 1, \operatorname{PPAR} \gamma$, and the proliferation marker Ki67. Primary and secondary antibodies were used at concentrations of 1:100. Leftmost panel shows hematoxylin and eosin staining (H\&E) staining of the tumor and cerebellar molecular layer $(\mathrm{mol})$ and inner granule layer $(I G L)$. The non-tumorous cerebellum immunofluorescence focuses on the boundary between the IGL and molecular layer demonstrated in the H\&E staining. Magnification: $\times 1.25$ (leftmost panel), $\times 40$ (other images). Bars $16 \mu \mathrm{M}$. Panels demonstrate the typical outcome from 5 different

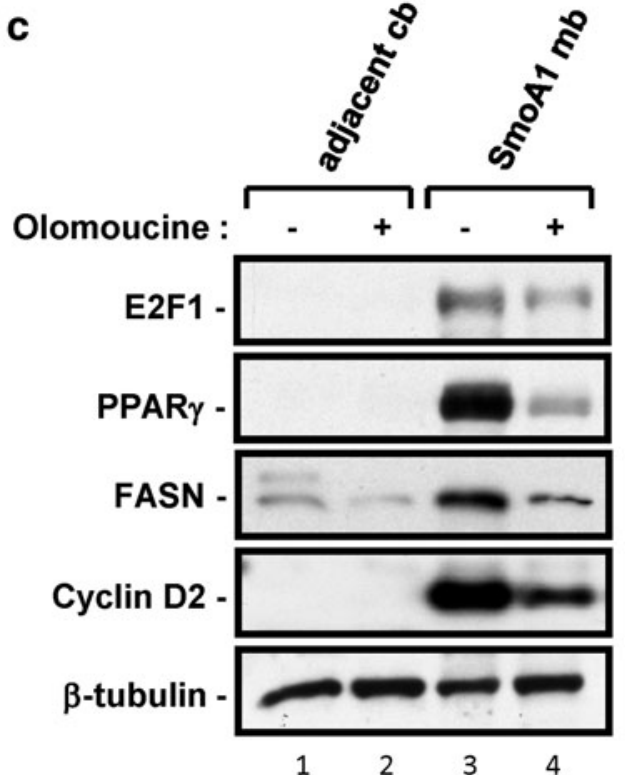

tumor and adjoining cerebellum specimens all paraffin embedded, sectioned, and subjected to immunofluorescence for the aforementioned antibodies. Additionally, the Oil red oil represents a typical outcome based on the staining of frozen sections from 5 different specimens. b Protein lysates were prepared from NeuroD2-SmoA1 medulloblastoma and normal cerebellum adjacent to the tumor, and then analyzed by western blotting for $\operatorname{PPAR} \gamma$, lipogenic marker (FASN) and proliferation markers (E2F1 and cyclin D2). $40 \mu \mathrm{g}$ protein/lane was loaded. Data presented are typical for all 5 sets of tumor and cerebellum samples that were studied. c Western blot analysis of proteins regulating proliferation and lipogenesis in adjacent non-tumor cerebellum or medulloblastomas from NeuroD2-SmoA1 mice treated with DMSO (-) or cdk inhibitor olomoucine $(+)$. The results shown represent a typical outcome amongst the control and experimental groups of mice 
we have also shown previously in that context that E2F1 is required for expression of the key lipogenic enzymes including fatty acid synthase (FASN) and acetyl CoA carboxylase (ACC), and that abrogation of E2F1 activity in vivo results in impaired lipogenesis in tumors. This firmly linked E2F1 activity downstream of Shh to de novo lipid synthesis in medulloblastoma and their proposed cells-oforigins.

PPAR $\gamma$ is a pivotal transcription factor that is triggered by fatty acid ligands and a key regulator of lipogenesis. Here, using immunostaining and blotting techniques in mouse NeuroD2-SmoA1 medulloblastomas, we detected very robust levels of PPAR $\gamma$ in the tumors as opposed to the adjacent non-tumor cerebellar tissues (Fig. 1a, b). We next examined whether in medulloblastomas expression of PPAR $\gamma$, like that of FASN, requires E2F1 activity. PPAR $\gamma$ has been reported to be transcriptionally controlled by E2F1 in adipocytes [7]. We treated medulloblastomabearing mice with the cdk inhibitor olomoucine $(6 \mathrm{mg} / \mathrm{kg})$ for 10 days. As we have previously reported, this treatment regimen markedly prolongs survival of tumor-bearing mice and is associated with reduced E2F1 and FASN expression (Fig. 1c) and with diminished proliferation in the tumors, as indicated by reduced levels of cyclin D2 (Fig. 1c). Indeed, olomoucine treatment resulted in a striking reduction of PPAR $\gamma$ levels in the NeuroD2-SmoA1 medulloblastomas (Fig. 1c). These results suggest that PPAR $\gamma$ lies downstream of the Shh $\rightarrow$ E2F1 axis in Shhdriven medulloblastomas and further, that investigating pathways downstream of PPAR $\gamma$ could lead to a better insight into the biology of medulloblastoma and their cancer associated metabolic patterns.

\section{Glycolytic enzymes are elevated in NeuroD2-SmoA1 medulloblastomas}

Substrate utilization pathways are functionally intertwined. Cells in anabolic tissues have a large capacity to generate anew and store fats from excessive glucose influx. We have shown that Shh-driven medulloblastomas and their proposed cells-of-origin, cerebellar granule neuron precursors (CGNPs), exhibit high levels of lipogenesis, and here we report that these tumors feature increased PPAR $\gamma$. In order to determine whether glycolysis is increased in NeuroD2SmoA1 medulloblastomas, we carried out immunostaining and western blot analysis of medulloblastomas and adjacent, non-tumorous cerebellum. As shown in Fig. 2a, b, the medulloblastomas are marked by a robust accumulation of many key glycolytic enzymes, including hexokinase II (HKII), pyruvate kinase M2 (PKM2), and glucose transporter type 4 (Glut4), which control glucose transport, especially into muscle and adipocytes in response to insulin stimulation. Notably such patterns appear to be specific to
HKII as hexokinase I (HKI) levels were higher in normal cerebellar tissue than in the medulloblastomas.

We next asked whether the expression of these glycolytic enzymes, like that of lipogenic markers, is dependent upon $\mathrm{Rb}$ inactivation and E2F1 activity. To this end we used western blot analysis of cerebellar tissue and medulloblastomas prepared from DMSO- or olomoucine-treated mice. As shown in Fig. 2c, levels of HKII, PKM2, and Glut4 were all reduced in olomoucine-treated tumors, while levels of HKI were up-regulated in the olomoucinetreated medulloblastomas. These results indicate that the glycolytic markers are associated with E2F1 activity and proliferation, and further suggest that HKI plays a role distinct from glycolysis in differentiated (e.g. normal cerebellum) or non-proliferating tissue (e.g. olomoucinetreated medulloblastoma).

E2F1 is required for PPAR $\gamma$ and the glycolytic markers expression in neural precursors of the developing cerebellum

Medulloblastomas associated with increased activity of the Shh pathway have been proposed to arise from proliferating neural precursors in the developing cerebellum [6]. During perinatal development, these cells proliferate rapidly in the external germinal layer (EGL) response to Shh secreted from the underlying Purkinje cells (PN) [4, 44, 45]. Upon exiting the cell cycle they migrate inward to the internal granule layer (IGL), where they undergo terminal differentiation. Shh-associated medulloblastomas share many characteristics of these cerebellar granule neuron precursors (CGNPs), including high expression of $\mathrm{N}$-myc, microRNA miR 17/92, increased lipogenesis, and as we previously reported and show again here (Fig. 3a, b) elevated E2F1 [2, 29, 33]. To determine whether increased glycolysis is also a shared feature of Shh-associated medulloblastomas and CGNPs, and whether this is, like lipogenesis, an E2F1-dependent event, we carried out immunofluorescence and western blot analysis of wild-type and E2F1-null mouse cerebella at postnatal day 7 (PN7), when CGNP proliferation is at its peak.

As shown in Fig. 3a (bottom panels) and in Fig. 3b, PPAR $\gamma$ is found in the EGL, as are HKII, PKM2, and Glut4. We also observed some expression of Glut4 in the IGL. In contrast, HKI was absent from the EGL and was highly expressed in the IGL, where differentiating CGNPs are found. Ablation of E2F1 resulted in loss of expression of PPAR $\gamma$, HKII, PKM2, and Glut4, but did not affect levels of HKI. These results suggest that in the developing cerebellum, as in medulloblastoma, $\operatorname{PPAR} \gamma$ and glycolytic markers lie downstream of Sonic hedgehog and E2F1.

We next asked whether CGNPs carry out glycolysis in vitro, and whether acute manipulation of E2F1 abrogates 

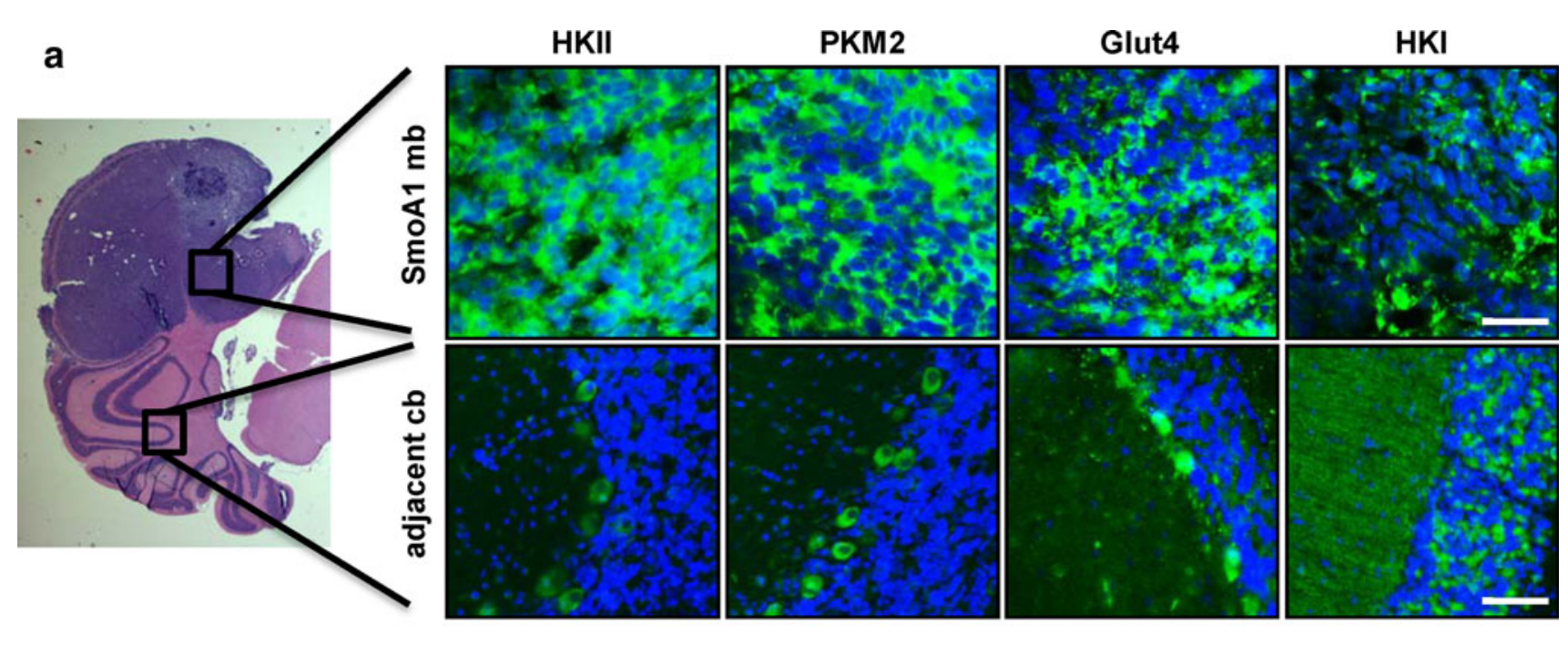

b

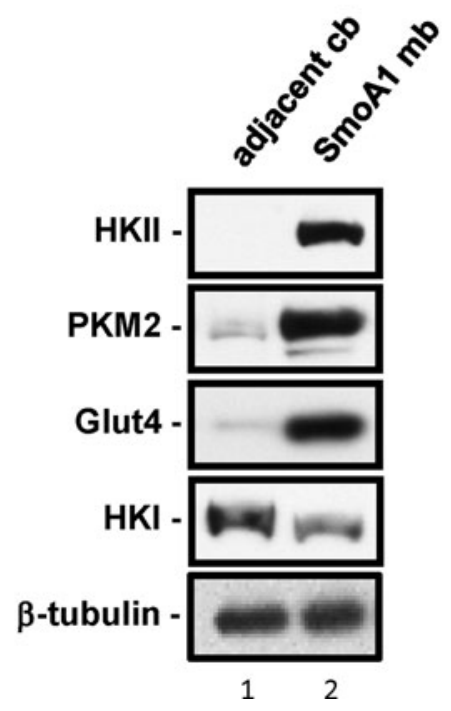

Fig. 2 Shh-induced mouse medulloblastomas have high levels of glycolytic markers of hexokinase II, pyruvate kinase M2, and glucose transporter-4. a NeuroD2-SmoA1 medulloblastoma (top row) and adjacent non-tumor cerebellum (bottom row) were subjected to immunofluorescence analyses for glycolysis (HKI, HKII, Glut4, and PKM2). Leftmost panel shows hematoxylin and eosin staining (H\&E) staining of the tumor and cerebellar molecular layer $(\mathrm{mol})$ and inner granule layer $(I G L)$. Magnification: $\times 1.25$ (leftmost panel), $\times 40$ (other images). Bars $16 \mu \mathrm{M}$. Results shown are typical for the 5 different tumor and adjoining cerebellum samples used for immunofluorescence in this study. b Protein lysates were prepared from

this, as it does lipogenesis [2]. We established primary cultures of CGNPs from PN 4/5 neonatal mice. These cells are grown in the absence of serum; addition of exogenous Shh to their medium promotes ongoing proliferation, while in its absence the cells leave the cell cycle and differentiate as they would in vivo. We infected Shh-treated CGNPs

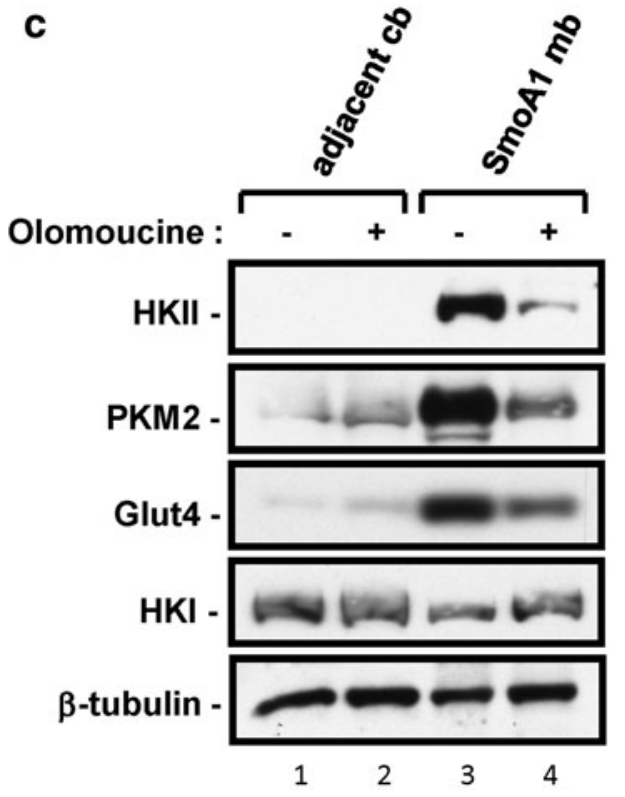

NeuroD2-SmoA1 medulloblastoma and normal cerebellum adjacent to the tumor, and then analyzed by western blotting for glycolysis. $40 \mu \mathrm{g}$ protein/lane was loaded. The data shown were repeated with multiple sets of tumor and cerebellum from different NeuroD2SmoA1 mice exhibiting tumors. c Western blot analysis of proteins regulating glycolysis in adjacent non-tumor cerebellum or medulloblastomas from NeuroD2-SmoA1 mice treated with DMSO (-) or $\mathrm{cdk}$ inhibitor olomoucine $(+)$. The same protein lysates from Fig. 1c were blotted for glycolytic markers and this is a typical outcome of those blots

with lentiviruses carrying a control, scrambled short hairpin RNA (shRNA) sequence or a shRNA sequence targeting E2F1 for knock-down. As shown in Fig. 3c and as previously reported, these lentiviruses effectively knocked down E2F1, while infection with the control shRNA had no effect on E2F1 levels. In the presence of 


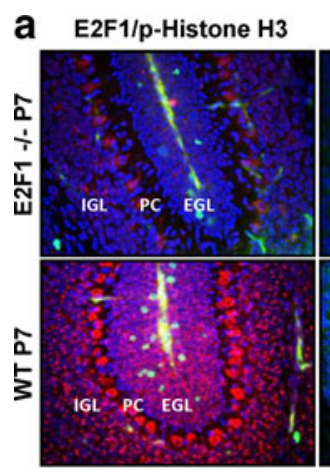

b

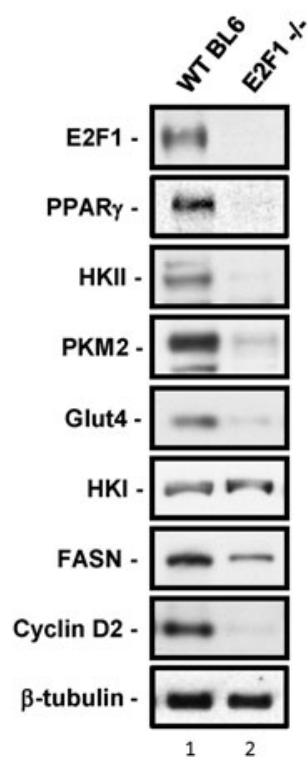

Fig. 3 Loss of E2F1 in mice ablates PPAR $\gamma$, and key markers of de novo lipogenesis, glycolysis, and cell proliferation. a PN 7 sagittal sections of wild-type (bottom row) and E2F1-null (top row) cerebella were immunostained for the glycolysis-related proteins Glut4, muscle pyruvate kinase 2 (PKM2), hexokinase 1 and 2 (HKI; HKII) as well as PPAR $\gamma$, and E2F1 with the proliferation marker phospho-histone H3. First column panel shows cerebellar inner granule layer $(I G L)$, Purkinje cells $(P C)$, and external granule layer $(E G L)$. Magnification: $\times 40$. Bars $32 \mu \mathrm{M}$. These slides are indicative of several specimens from the wildtype and E2F null groups that were analyzed with IF.

Shh, CGNPs have increased levels of PPAR $\gamma$, HKII, PKM2, and Glut4, consistent with our observations in vivo. Levels of HKI were unaffected by the presence of Shh. Infection with the scrambled shRNA results in a slight reduction of PPAR $\gamma$ levels but has no effect on levels of the glycolytic markers. In contrast, E2F1 knock-down effectively abrogated expression of PPAR $\gamma$ and the associated glycolytic markers. Taken together, the results of our in vivo analysis and in vitro manipulation of E2F1 indicate that in Shh-associated mouse medulloblastomas and in proliferating CGNPs, glycolysis is elevated in an E2F1dependent manner and its regulation functionally lie downstream of PPAR $\gamma$.
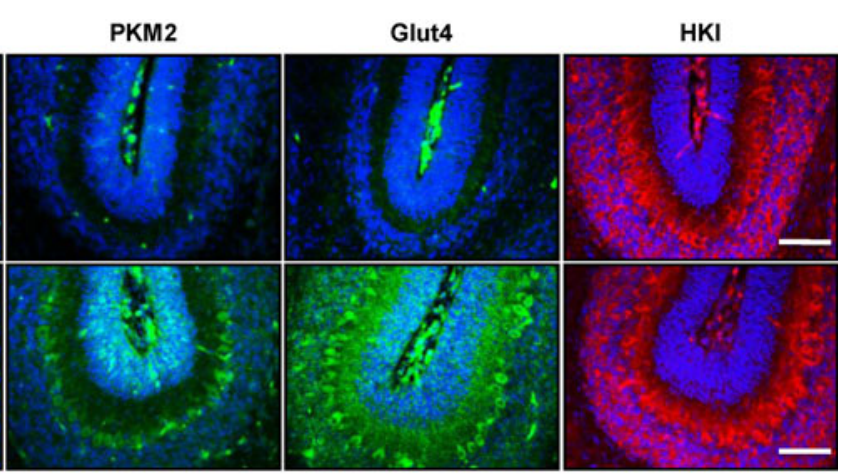

C

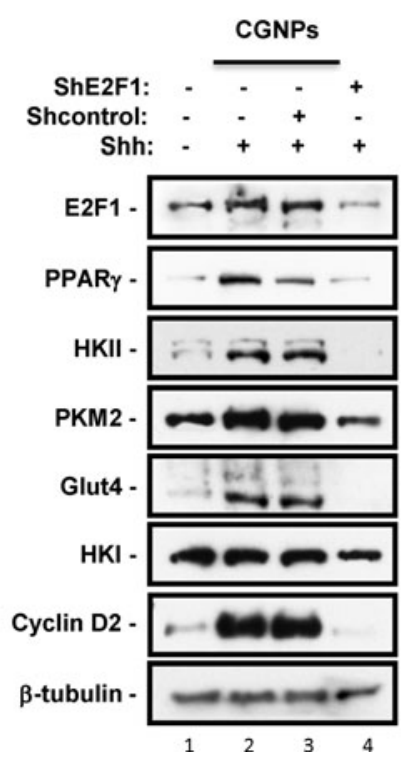

b Cerebella were collected from wild-type or E2F1-null PN 5 pups, then analyzed by western blotting for levels of E2F1, PPAR $\gamma$, glycolysis proteins (HKI, HKII, PKM2, and Glut4), the lipogenic marker fatty acid synthase (FASN), and proliferation marker cyclin D2. Results are typical of several sets of protein lysates that were collected. c Western blot analysis of E2F1, PPAR $\gamma$, glycolysis (HKI, HKII, PKM2, Glut4) and proliferation marker cyclin D2 in CGNPs treated with DMSO vehicle, Shh or Shh in the presence of lentiviruses carrying shRNAs targeting E2F1 or a scrambled control shRNA virus

PPAR $\gamma$ activity is required for the expression of glycolytic markers in CGNPs

CGNPs require Shh pathway activity for proliferation as well as for E2F1 expression. To establish that PPAR $\gamma$ and glycolysis are regulated by Shh upstream of E2F1, we cultured CGNPs in the presence of Shh or in the presence of Shh and cyclopamine, which inhibits Smoothened and blocks Shh signaling. As we have shown previously, cyclopamine treatment blunts the accumulation of E2F1 and FASN, and reduced proliferation as determined by cyclin D2 levels (Fig. 4a). Exposure to cyclopamine had no effect on HKI. However, in the presence of cyclopamine 
levels of PPAR $\gamma$, HKII, PKM2, and Glut4 were strongly reduced, consistent with Shh signaling being required for E2F1 activation and induction of glycolysis, and indicating that glycolysis is a property associated with CGNP proliferation.

Thus far, our results indicate a requirement for E2F1 in the regulation of PPAR $\gamma$ and the enzymes promoting glycolysis. As shown in Fig. 3, PPAR $\gamma$ and glycolytic enzymes lie downstream of E2F1. We wished to determine whether the activity of PPAR $\gamma$ is required for glycolytic marker up-regulation in CGNPs. It is known that treatment of cells with PPAR $\gamma$ agonists such as rosiglitazone is associated with induction of glycolytic enzymes including Glut4 [8], although the precise mechanism through which this takes place is unknown. We carried out dose-response studies with the PPAR $\gamma$-specific inhibitor GW9662, which has been used in vitro and in vivo to inhibit PPAR $\gamma$ and displays no activity towards other PPAR family members $[23,28]$. As shown in Fig. 4b, treatment of CGNPs with 50 or $100 \mathrm{nM} \mathrm{GW-9662} \mathrm{resulted} \mathrm{in} \mathrm{loss} \mathrm{of} \mathrm{detectable} \mathrm{PPAR} \gamma$, which was remarkably associated with reduced HKII, PKM2, and Glut4. Importantly, GW9662 treatment had no effect on HKI and also did not reduce levels of FASN, indicating that although both FASN and PPAR $\gamma$ lie downstream of E2F1, PPAR $\gamma$ and FASN are regulated independently. Notably, GW9662 treatment also reduced CGNP proliferation as indicated by diminished cyclin D2 levels. Thus, PPAR $\gamma$ activity is required for glycolysis and ongoing cell division in CGNPs.

Inhibition of PPAR $\gamma$ impairs glycolysis in medulloblastoma and prolongs survival of tumor-bearing mice

Our data in CGNPs show that PPAR $\gamma$ is associated with a proliferative state, and that PPAR $\gamma$ positively regulates glycolytic markers. Many tumor cells are known to exhibit high levels of glycolysis, and NeuroD2-SmoA1 medulloblastomas are consistently highly proliferative. We wished to determine whether PPAR $\gamma$ inhibition could impair
Fig. 4 Glycolysis in CGNPs is Shh- and PPAR $\gamma$-dependent. a CGNP cultures were prepared for western blot analysis from PN 4/5 mice and incubated with vehicle (Veh), Shh $(3 \mu \mathrm{g} / \mathrm{ml})$ and/or Smoothened inhibitor cyclopamine $(10 \mu \mathrm{g} / \mathrm{ml})$. The lysates were blotted for the glycolytic and proliferative proteins. The presented work was repeated with several sets of CGNP lysates from PN 4/5 mice. b CGNP cultures were prepared for western blot analysis from PN 4/5 mice and incubated with vehicle (Veh), Shh $(3 \mu \mathrm{g} / \mathrm{ml})$ and/or PPAR $\gamma$ antagonist GW9662 in increasing doses of 0,50 , and $100 \mathrm{nM}$
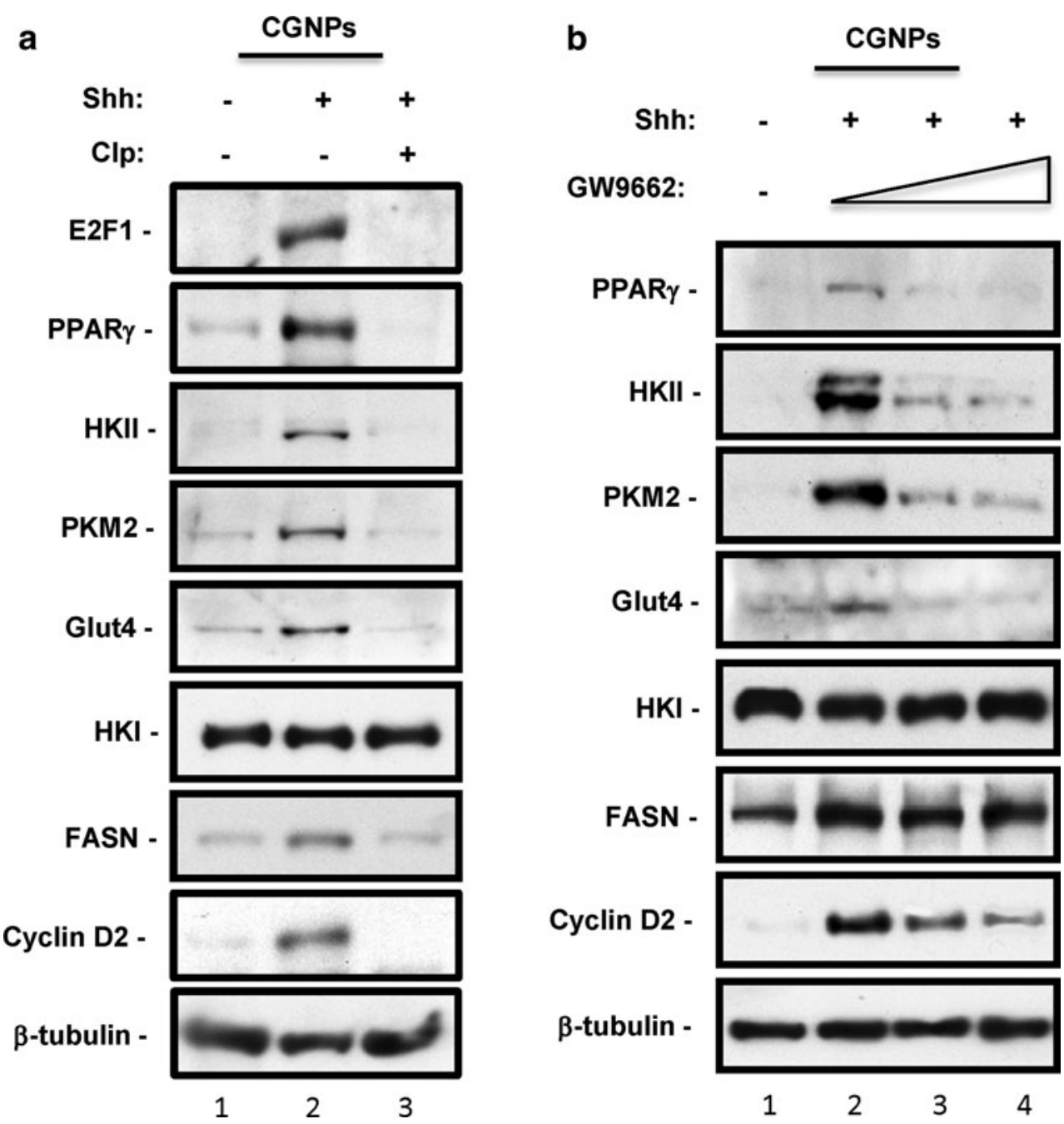

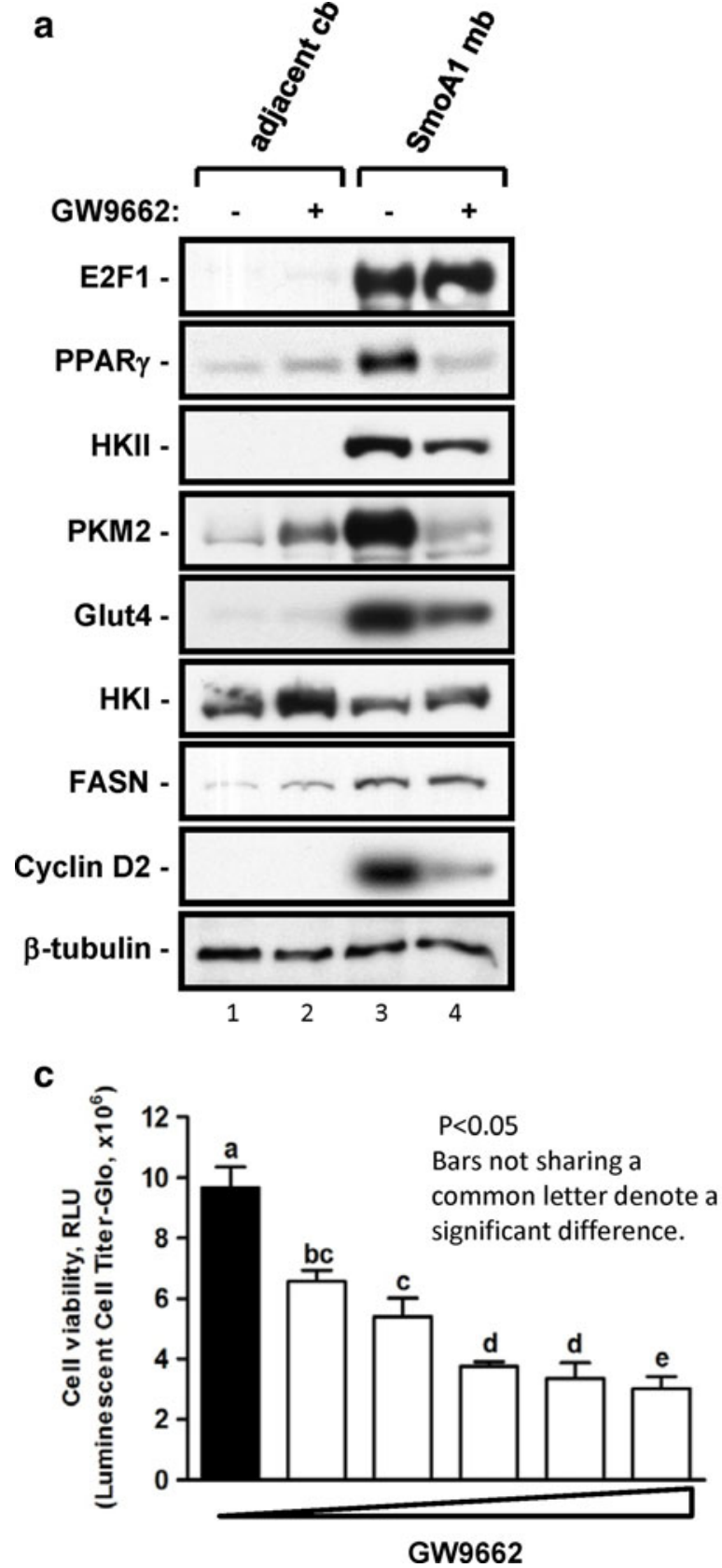

Fig. 5 Treatment of medulloblastoma-bearing mice with PPAR $\gamma$ antagonist GW9662 impairs glycolysis in tumor in vivo. a Western blot analysis for E2F1, PPAR $\gamma$, glycolysis (HKI, HKII, PKM2, and Glut4), lipid synthesis (FASN) and proliferation (cyclin D2) in samples of adjacent cerebella and medulloblastomas from vehicle (DMSO)treated (-) or GW9662-treated (+) mice. The results shown are typical for several control and experimental sets of mice. b Immunostaining in adjacent cerebellum and medulloblastomas in vehicle- or GW9662treated mice. Slides from the tissue of several untreated and treated

glycolysis and affect proliferation in these tumors. We treated medulloblastoma-bearing NeuroD2-SmoA1 mice with GW9662 or its vehicle DMSO for up to 10 days, then harvested the tumors for western blot and

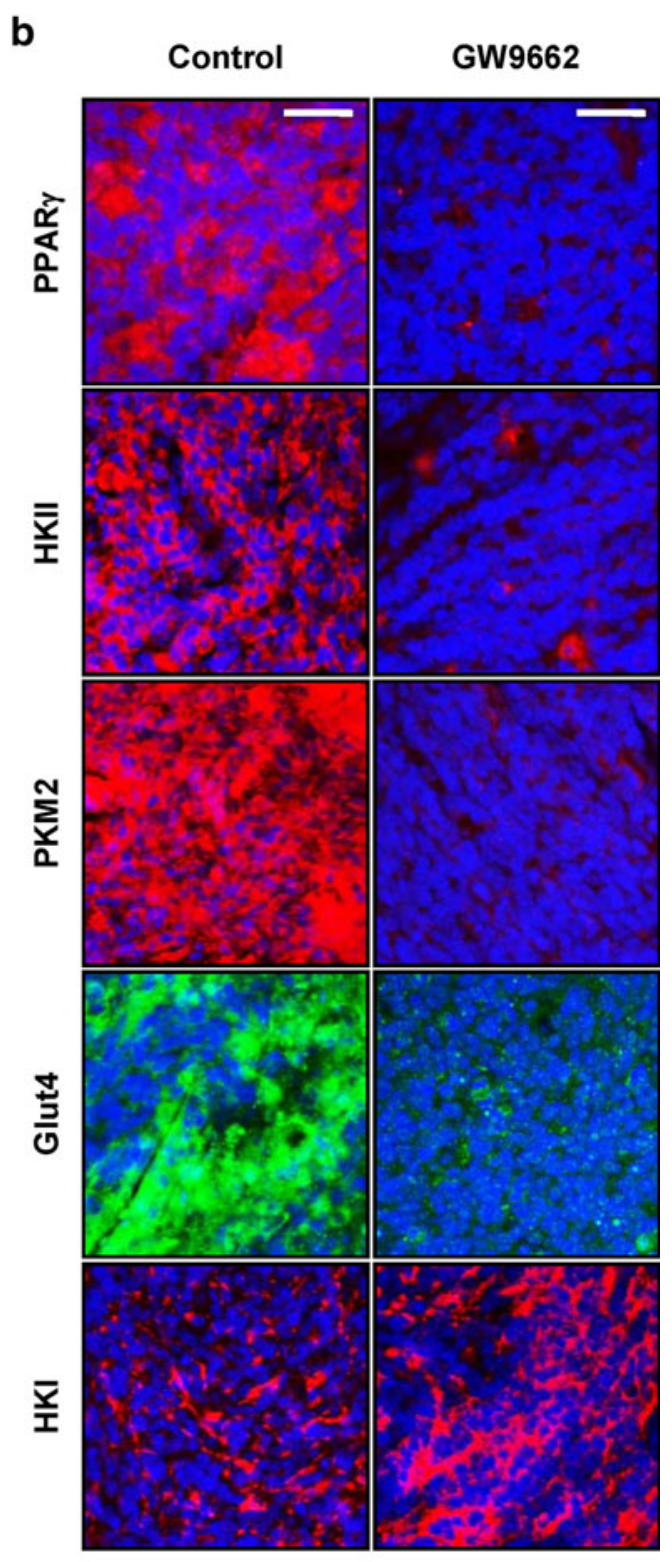

NeuroD2-SmoA mice exhibiting medulloblastomas were analyzed and the presented results were typical. Magnification, $\times 40$. Bars $16 \mu \mathrm{M}$. c Effects of increasing doses of the PPAR $\gamma$ antagonist GW9662 $(0,0.01$, $0.02,0.05,0.07,0.1 \mu \mathrm{M}$ ) for $24 \mathrm{~h}$ on pzp53med cell viability as determined by cell Titer-Glo assay, a bioluminescent analysis based on the presence of ATP. Each bar in the bioluminescence graph represents the average of separate quadruplicate determinations with error bars showing the standard deviation of the mean. Bars not sharing common letter are significantly different at $P<0.05$

immunofluorescence analysis. As shown in Fig. 5a, GW9662 treatment had no impact on the level of E2F1 or FASN, but sharply reduced PPAR $\gamma$ protein levels (Fig. 5a, b), consistent with PPAR $\gamma$ lying downstream of E2F1 and in a 
parallel pathway to FASN. GW9662 treatment also reduced levels of glycolytic markers and cyclin D2 protein, but had no effect on HKI, in keeping with our in vitro results in CGNPs. When tested in the pzp53 MB cell line, GW9662 treatment was very effective in killing cancer cells in a dose-dependent manner (Fig. 5c), as show by a luminescence viability assay (Cell Titer-Glo).

Using FDG-PET analysis, we observed a striking reduction in glucose uptake in GW9662-treated mice (Fig. 6a). This correlates with a functional effect of PPAR $\gamma$ inhibition on de novo lipid synthesis (Fig. 6b). Finally, we

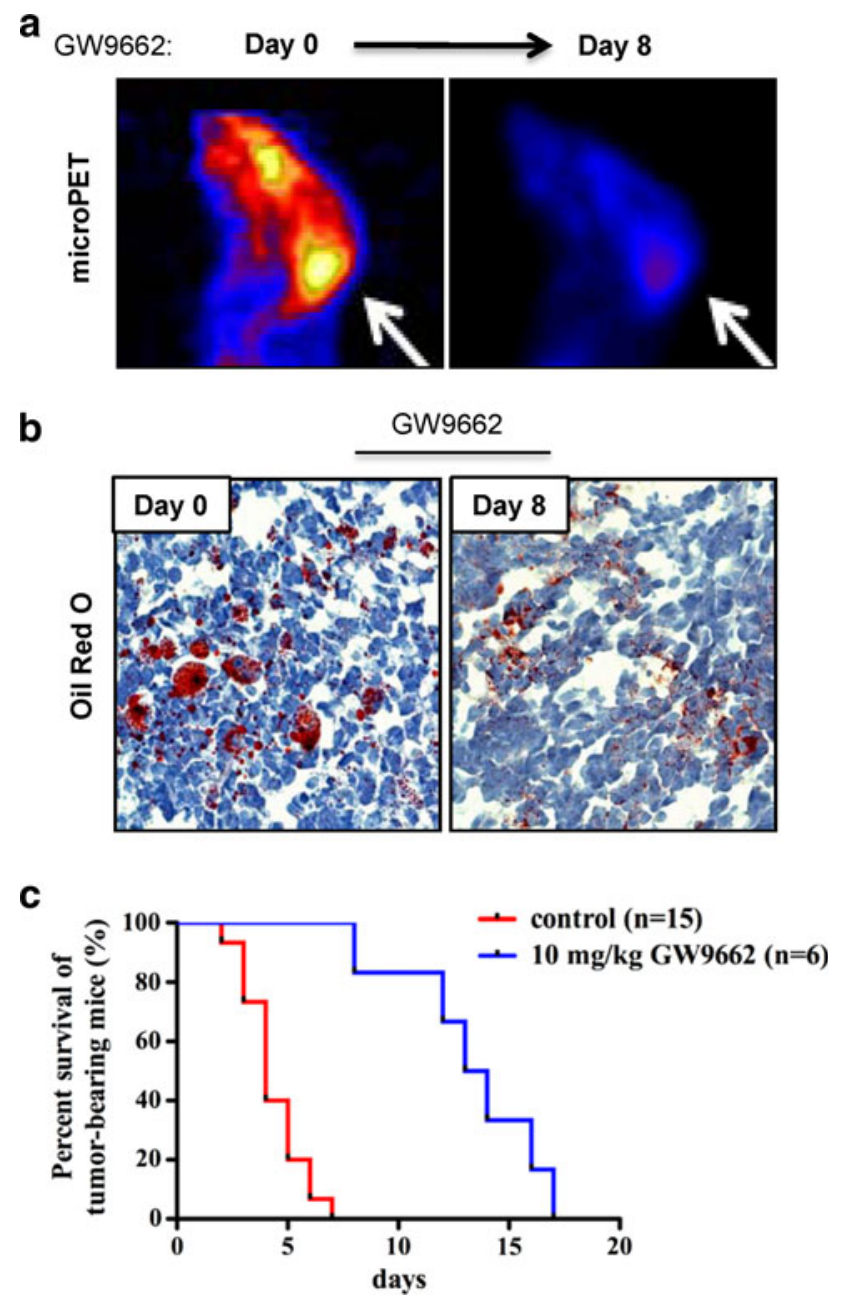

Fig. 6 Treatment of medulloblastoma-bearing mice with PPAR $\gamma$ antagonist GW9662 prolongs survival and reduces glucose uptake in vivo. a FDG-PET scan of medulloblastoma-bearing NeuroD2-SmoA1 mouse before and after treatment with the PPAR $\gamma$ antagonist GW9662 (10 mg/kg, intraperitoneal injection daily over the 8-study day period). b A representative Oil red O staining of medulloblastoma sections from an untreated mouse (day 0$)$ and a counterpart treated with GW9662 for 8 days. c Kaplan-Meyer survival curve of NeuroD2-SmoA1 medulloblastoma-bearing mice in days commencing with initiation of treatment with DMSO or GW9662.; $* P$ value $<0.0001$, Mantel-Cox test analysis). Magnification, $\times 40$. Bars $16 \mu \mathrm{M}$ assayed the effects of GW9662 treatment on survival of NeuroD2-SmoA1 medulloblastoma-bearing mice. As shown in Fig. 6c, from the time of tumor onset as determined by appearance of detectable symptoms such as head tilt and altered gait and confirmed by magnetic resonance imaging, the average length of survival of NeuroD2-SmoA1 animals treated with DMSO $(N=15)$ was approximately a week. In contrast, GW9662-treated mice $(N=6)$ lived for up to 16 days after tumor onset, a significant increase in survival. These striking results suggest a therapeutic potential for PPAR $\gamma$ inhibition in treating Shh-driven medulloblastomas.

\section{Discussion}

Cross talk between independent yet intertwined signaling pathways of metabolism and cancer is currently the topic of increasing scrutiny. We reported earlier a direct transcriptional link between the $\mathrm{Rb} / \mathrm{E} 2 \mathrm{~F}$ tumor suppressor complex and PDK4 [17], a critical nutrient sensor and a regulator of mitochondrial glucose oxidation. In more recent work, we demonstrated that excessive mitogenic signaling by Shh, a driver of medulloblastoma, engages the $\mathrm{Rb} / \mathrm{E} 2 \mathrm{~F}$ tumor suppressor complex, activates the key enzyme fatty acid synthase (FASN) and drives lipogenesis through a novel, E2F1-regulated process [2]. FASN has also been linked to survival in glioma cells with activating EGFR mutations [11, 12] and other tumors [25]. Many transformed cells also exhibit reliance on glutaminolysis [5, 43], a process associated with the activity of the myc oncogene [9, 46]. In addition, expression of key tumor suppressors such as p53, PTEN and LKB1 has been reported to influence important regulators of cellular metabolism [18]. Indeed, many signaling pathways implicated in routine surveillance themselves go awry across the spectrum of human diseases. Loss of metabolic inflexibility for instance is a pathological feature in disorders like diabetic cardiomyopathy as well as in certain cancers, where tumor cells' addiction to de novo lipid synthesis for instance appears to be a defining feature for proliferation and survival [2, 24].

In this study, we show that in medulloblastoma and CGNPs, the Shh pathway is coupled-through E2F1-to the steroid receptor PPAR $\gamma$ and the control of glycolysis, a vital cellular process. This coupling underscores the broad influence of the hedgehog-regulated metabolic network. It is significant given the role of PPAR $\gamma$ as a modulator of cellular homeostasis as well as a therapeutic target in many chronic diseases [26]. Indeed, $\operatorname{PPAR} \gamma$ agonists are routinely used for the treatment of patients with diabetes or hyperglycemia and it is established that activation of PPAR $\gamma$ ameliorates insulin sensitivity in vivo and ex vivo. Nonetheless, the findings of this study reveal that 
antagonizing, not stimulating, PPAR $\gamma$ would have a practical therapeutic use in MBs. Indeed, inhibiting PPAR $\gamma$ was markedly beneficial in counteracting hyperproliferation, reducing tumor burden, and extending the survival of Shhdriven MBs in mice (Fig. 6). Of note, when examined for their PPAR $\gamma$ expression using tissue microarrays, marked PPAR $\gamma$ staining was detected in $>50 \%$ of the Shh subgroup (Supplementary data). Intriguingly high PPAR $\gamma$ staining was also present in other groups and prevalent in Group D samples. While more elaborate analysis (including demographic, gender, survival) in larger cohorts is needed to understand the role and function of PPAR $\gamma$ in human tumors, deregulation of this pathway appears to be widespread in human MB.

The aforementioned role for the E2F1 $\rightarrow$ PPAR $\gamma$ axis as a potent effector of hedgehog signaling in modulating the glycolytic index has also numerous implications. In addition to presenting a viable therapeutic avenue in this pediatric brain tumor, it helps explain tumor etiologies associated with hedgehog and/or $\mathrm{Rb} / \mathrm{E} 2 \mathrm{~F}$ deregulation. Elevated glucose uptake and hexokinase II activity have been characteristic features correlated with poor prognosis in a wide spectrum of malignancies. These features are routinely exploited in diagnostic imaging techniques like fluorodeoxyglucose (FDG)-positron emission tomography [13]. Yet, a sizable number of human cancers are also FDGnegative and a gradation of glycolic indices is reported within many tumor types that may or may not reflect specific lesions. Some positive regulators of cell proliferation are associated with reduced hexokinase activities and diminished glycolysis. For instance, antisense-mediated cyclin D1 down-modulation has been shown to upregulate glycolysis in vivo, by releasing hexokinase II from inhibition [38]. These contradictions perhaps reflect the intricacies underlying the cancer-associated metabolic networks and their confounding heterogeneity. In medulloblastoma, a unique, albeit more defined, metabolic pattern controlled by Shh is beginning to emerge: The striking accumulation of lipid droplets we describe earlier ([2] and Fig. 1a), is matched by the equally striking glycolytic index and confirmed by elevated FDG-PET activity. This phenotype is consistent with substrate utilization patterns in highly anabolic tissues where excessive glucose uptake is routed to fat production and excessive lipogenesis. Moreover, this particular substrate utilization pattern in shhdriven MBs unravels a new circuitry coupling hedgehog signaling directly to glucose uptake. At the heart of this process is the $\mathrm{Rb} / \mathrm{E} 2 \mathrm{~F}$ complex, itself the target of growth factors and external trophic signals and a tumor suppressor broadly inactivated in human cancers. Our work identified the (Shh $\dashv \mathrm{Rb} \dashv \mathrm{E} 2 \mathrm{~F} 1 \rightarrow$ PPAR) axis as a functional metabolic driver of medulloblastoma tumor formation and the core of its nutrient sensing network coupling external dietary conditions to the metabolic adaptation of the cellular milieu, including the regulation of the glycolic index. We note that in CGNPs, the mere incubation of primary cells with Shh markedly triggers Glut4-a glucose transporter typically induced with insulin stimulation. As such, glucose utilization in neural precursors could be primarily a Shhregulated event, irrespective of hormonal status. Indeed, our data implicate this E2F1-dependent glycolytic process as a systematic, distinctly cell-autonomous process that is mediated by PPAR $\gamma$ but triggered by Shh in medulloblastoma.

Acknowledgments This Study is supported by funding from the Qatar Foundation, Qatar National Research Fund (Z.N, NPRP 09-413-3-104) and the NINDS (A.M.K R01 NS061070). We thank Gary Sulikowski and Sean DeGuire (both at Vanderbilt University) for helpful discussions. We also would like to acknowledge the MSKCC Molecular Cytology Core Facility for their assistance in Oil Red O staining and the MSKCC Medical Physics core for their assistance in FDG-PET imaging.

Conflict of interest The authors declare no conflict of interest.

Open Access This article is distributed under the terms of the Creative Commons Attribution License which permits any use, distribution, and reproduction in any medium, provided the original author(s) and the source are credited.

\section{References}

1. Berman DM, Karhadkar SS, Hallahan AR, Pritchard JI, Eberhart CG, Watkins DN, Chen JK, Cooper MK, Taipale J, Olson JM, Beachy PA (2002) Medulloblastoma growth inhibition by hedgehog pathway blockade. Science 297(5586):1559-1561

2. Bhatia B, Hsieh M, Kenney AM, Nahle Z (2011) Mitogenic Sonic hedgehog signaling drives E2F1-dependent lipogenesis in progenitor cells and medulloblastoma. Nature 30(4):410-422. doi:10.1038/onc.2010.454

3. Bhatia B, Northcott PA, Hambardzumyan D, Govindarajan B, Brat DJ, Arbiser JL, Holland EC, Taylor MD, Kenney AM (2009) Tuberous sclerosis complex suppression in cerebellar development and medulloblastoma: separate regulation of mammalian target of rapamycin activity and p27 Kip1 localization. Cancer Res 69(18):7224-7234. doi:10.1158/0008-5472.CAN-09-1299

4. Dahmane N, Ruiz i Altaba A (1999) Sonic hedgehog regulates the growth and patterning of the cerebellum. Development 126(14):3089-3100

5. DeBerardinis RJ, Mancuso A, Daikhin E, Nissim I, Yudkoff M, Wehrli S, Thompson CB (2007) Beyond aerobic glycolysis: transformed cells can engage in glutamine metabolism that exceeds the requirement for protein and nucleotide synthesis. Proc Natl Acad Sci USA 104(49):19345-19350

6. Eberhart CG (2008) Even cancers want commitment: lineage identity and medulloblastoma formation. Cancer Cell 14(2):105107. doi:10.1016/j.ccr.2008.07.011

7. Fajas L, Landsberg RL, Huss-Garcia Y, Sardet C, Lees JA, Auwerx J (2002) E2Fs regulate adipocyte differentiation. Dev Cell 3(1):39-49

8. Festuccia WT, Blanchard PG, Turcotte V, Laplante M, Sariahmetoglu M, Brindley DN, Deshaies Y (2009) Depot-specific effects of the PPARgamma agonist rosiglitazone on adipose tissue glucose uptake and metabolism. J Lipid Res 50(6):11851194. doi:10.1194/jlr.M800620-JLR200 
9. Gao P, Tchernyshyov I, Chang TC, Lee YS, Kita K, Ochi T, Zeller KI, De Marzo AM, Van Eyk JE, Mendell JT, Dang CV (2009) c-Myc suppression of miR-23a/b enhances mitochondrial glutaminase expression and glutamine metabolism. Nature 458(7239):762-765. doi:10.1038/nature07823

10. Goodrich LV, Milenkovic L, Higgins KM, Scott MP (1997) Altered neural cell fates and medulloblastoma in mouse patched mutants. Science 277(5329):1109-1113

11. Guo D, Hildebrandt IJ, Prins RM, Soto H, Mazzotta MM, Dang J, Czernin J, Shyy JY, Watson AD, Phelps M, Radu CG, Cloughesy TF, Mischel PS (2009) The AMPK agonist AICAR inhibits the growth of EGFRvIII-expressing glioblastomas by inhibiting lipogenesis. Proc Natl Acad Sci USA 106(31):12932-12937. doi: 10.1073/pnas.0906606106

12. Guo D, Prins RM, Dang J, Kuga D, Iwanami A, Soto H, Lin KY, Huang TT, Akhavan D, Hock MB, Zhu S, Kofman AA, Bensinger SJ, Yong WH, Vinters HV, Horvath S, Watson AD, Kuhn JG, Robins HI, Mehta MP, Wen PY, DeAngelis LM, Prados MD, Mellinghoff IK, Cloughesy TF, Mischel PS (2009) EGFR signaling through an Akt-SREBP-1-dependent, rapamycin-resistant pathway sensitizes glioblastomas to antilipogenic therapy. Sci Signal 2 (101):ra82. doi:10.1126/scisignal.2000446

13. Haberkorn U, Markert A, Mier W, Askoxylakis V, Altmann A (2011) Molecular imaging of tumor metabolism and apoptosis. Oncogene 30(40):4141-4151. doi:10.1038/onc.2011.169

14. Hallahan AR, Pritchard JI, Hansen S, Benson M, Stoeck J, Hatton BA, Russell TL, Ellenbogen RG, Bernstein ID, Beachy PA, Olson JM (2004) The SmoA1 mouse model reveals that notch signaling is critical for the growth and survival of sonic hedgehog-induced medulloblastomas. Cancer Res 64(21):7794-7800

15. Hatton BA, Villavicencio EH, Tsuchiya KD, Pritchard JI, Ditzler S, Pullar B, Hansen S, Knoblaugh SE, Lee D, Eberhart CG, Hallahan AR, Olson JM (2008) The Smo/Smo model: hedgehoginduced medulloblastoma with $90 \%$ incidence and leptomeningeal spread. Cancer Res 68(6):1768-1776. doi:10.1158/00085472.CAN-07-5092

16. Hernando E, Nahle Z, Juan G, Diaz-Rodriguez E, Alaminos M, Hemann M, Michel L, Mittal V, Gerald W, Benezra R, Lowe SW, Cordon-Cardo C (2004) Rb inactivation promotes genomic instability by uncoupling cell cycle progression from mitotic control. Nature 430(7001):797-802. doi:10.1038/nature02820 nature 02820

17. Hsieh MCF, Das D, Sambandam N, Zhang MQ, Nahle Z (2008) Regulation of the PDK4 isozyme by the Rb-E2F1 complex. J Biol Chem 283(41):27410-27417

18. Jones RG, Thompson CB (2009) Tumor suppressors and cell metabolism: a recipe for cancer growth. Genes Dev 23(5):537548. doi:10.1101/gad.1756509

19. Kenney AMCM, Rowitch DH (2003) Nmyc upregulation by sonic hedgehog signaling promotes proliferation in developing cerebellar granule neuronal precursors. Development 130:15-28

20. Kenney AM, Rowitch DH (2000) Sonic hedgehog promotes G(1) cyclin expression and sustained cell cycle progression in mammalian neuronal precursors. Mol Cell Biol 20(23):9055-9067

21. Lau J, Hebrok M (2010) Hedgehog signaling in pancreas epithelium regulates embryonic organ formation and adult beta-cell function. Diabetes 59(5):1211-1221

22. Lavine K, Ornitz D (2009) Shared circuitry: developmental signaling cascades regulate both embryonic and adult coronary vasculature. Circ Res 104(2):159-169

23. Lee H, Finck BN, Jones LA, Welch MJ, Mach RH (2006) Synthesis and evaluation of a bromine-76-labeled PPARgamma antagonist 2-bromo-5-nitro- $N$-phenylbenzamide. Nucl Med Biol 33(7):847-854. doi:10.1016/j.nucmedbio.2006.08.003

24. Menendez JA (2009) Fine-tuning the lipogenic/lipolytic balance to optimize the metabolic requirements of cancer cell growth: molecular mechanisms and therapeutic perspectives. Biochim Biophys Acta. doi:10.1016/j.bbalip.2009.09.005

25. Menendez JA, Lupu R (2007) Fatty acid synthase and the lipogenic phenotype in cancer pathogenesis. Nat Rev Cancer 7(10):763-777. doi:10.1038/nrc2222

26. Nahle Z (2004) PPAR trilogy from metabolism to cancer. Curr Opin Clin Nutr Metab Care 7(4):397-402

27. Nahle Z, Polakoff J, Davuluri RV, McCurrach ME, Jacobson MD, Narita M, Zhang MQ, Lazebnik Y, Bar-Sagi D, Lowe SW (2002) Direct coupling of the cell cycle and cell death machinery by E2F. Nat Cell Biol 4(11):859-864. doi:10.1038/ncb868ncb868

28. Nakano R, Kurosaki E, Yoshida S, Yokono M, Shimaya A, Maruyama T, Shibasaki M (2006) Antagonism of peroxisome proliferator-activated receptor gamma prevents high-fat dietinduced obesity in vivo. Biochem Pharmacol 72(1):42-52. doi: 10.1016/j.bcp.2006.03.023

29. Northcott PA, Fernandez LA, Hagan JP, Ellison DW, Grajkowska W, Gillespie Y, Grundy R, Van Meter T, Rutka JT, Croce CM, Kenney AM, Taylor MD (2009) The miR-17/92 polycistron is up-regulated in sonic hedgehog-driven medulloblastomas and induced by $\mathrm{N}$-myc in sonic hedgehog-treated cerebellar neural precursors. Cancer Res 69(8):3249-3255. doi:10.1158/00085472.CAN-08-4710

30. Northcott PA, Korshunov A, Witt H, Hielscher T, Eberhart CG, Mack S, Bouffet E, Clifford SC, Hawkins CE, French P, Rutka JT, Pfister S, Taylor MD (2011) Medulloblastoma comprises four distinct molecular variants. J Clin Oncol 29(11):1408-1414. doi: 10.1200/JCO.2009.27.4324

31. Packer RJ, Cogen P, Vezina G, Rorke LB (1999) Medulloblastoma: clinical and biologic aspects. Neuro-oncol 1(3):232-250

32. Pietsch T, Waha A, Koch A, Kraus J, Albrecht S, Tonn J, Sorensen N, Berthold F, Henk B, Schmandt N, Wolf HK, von Deimling A, Wainwright B, Chenevix-Trench G, Wiestler OD, Wicking C (1997) Medulloblastomas of the desmoplastic variant carry mutations of the human homologue of Drosophila patched. Cancer Res 57(11):2085-2088

33. Pomeroy SL, Tamayo P, Gaasenbeek M, Sturla LM, Angelo M, McLaughlin ME, Kim JY, Goumnerova LC, Black PM, Lau C, Allen JC, Zagzag D, Olson JM, Curran T, Wetmore C, Biegel JA, Poggio T, Mukherjee S, Rifkin R, Califano A, Stolovitzky G, Louis DN, Mesirov JP, Lander ES, Golub TR (2002) Prediction of central nervous system embryonal tumour outcome based on gene expression. Nature 415(6870):436-442

34. Raffel C, Jenkins RB, Frederick L, Hebrink D, Alderete B, Fults DW, James CD (1997) Sporadic medulloblastomas contain PTCH mutations. Cancer Res 57(5):842-845

35. Rao G, Pedone CA, Coffin CM, Holland EC, Fults DW (2003) c-Myc enhances sonic hedgehog-induced medulloblastoma formation from nestin-expressing neural progenitors in mice. Neoplasia 5(3):198-204

36. Rao G, Pedone CA, Valle LD, Reiss K, Holland EC, Fults DW (2004) Sonic hedgehog and insulin-like growth factor signaling synergize to induce medulloblastoma formation from nestinexpressing neural progenitors in mice. Nature 23(36):61566162

37. Reifenberger J, Wolter M, Weber RG, Megahed M, Ruzicka T, Lichter P, Reifenberger G (1998) Missense mutations in SMOH in sporadic basal cell carcinomas of the skin and primitive neuroectodermal tumors of the central nervous system. Cancer Res 58(9):1798-1803

38. Sakamaki T, Casimiro MC, Ju X, Quong AA, Katiyar S, Liu M, Jiao X, Li A, Zhang X, Lu Y, Wang C, Byers S, Nicholson R, Link T, Shemluck M, Yang J, Fricke ST, Novikoff PM, Papanikolaou A, Arnold A, Albanese C, Pestell R (2006) Cyclin D1 determines mitochondrial function in vivo. Mol Cell Biol 26(14):5449-5469. doi:10.1128/MCB.02074-05 
39. Sugden MC, Bulmer K, Holness MJ (2001) Fuel-sensing mechanisms integrating lipid and carbohydrate utilization. Biochem Soc Trans 29(Pt 2):272-278

40. Sugden MC, Holness MJ (2006) Mechanisms underlying regulation of the expression and activities of the mammalian pyruvate dehydrogenase kinases. Arch Physiol Biochem 112(3):139-149

41. Sugden MC, Zariwala MG, Holness MJ (2009) PPARs and the orchestration of metabolic fuel selection. Pharmacol Res 60(3):141-150. doi:10.1016/j.phrs.2009.03.014

42. Taylor MD, Liu L, Raffel C, Hui CC, Mainprize TG, Zhang X, Agatep R, Chiappa S, Gao L, Lowrance A, Hao A, Goldstein AM, Stavrou T, Scherer SW, Dura WT, Wainwright B, Squire JA, Rutka JT, Hogg D (2002) Mutations in SUFU predispose to medulloblastoma. Nat Genet 31(3):306-310. doi:10.1038/ng916

43. Tong X, Zhao F, Thompson CB (2009) The molecular determinants of de novo nucleotide biosynthesis in cancer cells. Curr Opin Genet Dev 19(1):32-37. doi:10.1016/j.gde.2009.01.002

44. Wallace VA (1999) Purkinje-cell-derived Sonic hedgehog regulates granule neuron precursor cell proliferation in the developing mouse cerebellum. Curr Biol 9(8):445-448

45. Wechsler-Reya RJ, Scott MP (1999) Control of neuronal precursor proliferation in the cerebellum by Sonic Hedgehog. Neuron 22(1):103-114
46. Wise DR, DeBerardinis RJ, Mancuso A, Sayed N, Zhang XY, Pfeiffer HK, Nissim I, Daikhin E, Yudkoff M, McMahon SB, Thompson CB (2008) Myc regulates a transcriptional program that stimulates mitochondrial glutaminolysis and leads to glutamine addiction. Proc Natl Acad Sci USA 105(48):18782-18787. doi:10.1073/pnas.0810199105

47. Wu L (2001) The E2F1-3 transcription factors are essential for cellular proliferation. Nature 414(6861):325-329

48. Xie J, Johnson RL, Zhang X, Bare JW, Waldman FM, Cogen PH, Menon AG, Warren RS, Chen LC, Scott MP, Epstein EH Jr (1997) Mutations of the PATCHED gene in several types of sporadic extracutaneous tumors. Cancer Res 57(12):2369-2372

49. Xie J, Murone M, Luoh SM, Ryan A, Gu Q, Zhang C, Bonifas JM, Lam CW, Hynes M, Goddard A, Rosenthal A, Epstein EH Jr, de Sauvage FJ (1998) Activating Smoothened mutations in sporadic basal-cell carcinoma. Nature 391(6662):90-92

50. Yauch RL, Dijkgraaf GJ, Alicke B, Januario T, Ahn CP, Holcomb T, Pujara K, Stinson J, Callahan CA, Tang T, Bazan JF, Kan Z, Seshagiri S, Hann CL, Gould SE, Low JA, Rudin CM, de Sauvage FJ (2009) Smoothened mutation confers resistance to a hedgehog pathway inhibitor in medulloblastoma. Science. doi: 10.1126/science. 1179386 